Par la collation du doctorat honoris causa au professeur Cassese, l'Université de Genève, qui a contribué à sa formation et qu'il a servie en tant qu'enseignant, entend exprimer la profonde reconnaissance qui lui est due pour l'œuvre admirable d'une vie dépensée au service des idéaux de justice et d'humanité.

Faculté de dROIT de L’Université de Genève Janvier 2000

\title{
Konstantin Obradovic
}

$1939-2000$

Le professeur Konstantin Obradovic est décédé en mars de cette année. La nouvelle a frappé tous ses nombreux amis, qui voyaient en lui un éternel jeune homme, toujours prêt à s'enthousiasmer pour de bonnes causes, à se révolter contre l'injustice et la violence.

Konstantin Obradovic était un professeur reconnu et unanimement apprécié, qui a enseigné dans plusieurs universités en République fédérale de Yougoslavie, mais aussi dans de nombreuses universités étrangères. Ce qui frappait chez lui, c'était le souci de toujours aller au fond des choses, de ne pas se contenter de raisonnements juridiques de surface, satisfaisants pour l'esprit mais éloignés de la réalité. Cette attitude fut la sienne pour l'ensemble des problèmes internationaux, et tout particulièrement pour ceux relevant du droit international humanitaire, matière dont il était un des experts les plus connus et à laquelle il portait un attachement privilégié.

Konstantin Obradovic avait en effet participé à la Conférence diplomatique sur la réaffirmation et le développement du droit international humanitaire qui, à travers quatre sessions organisées de 1974 à 1977, avait élaboré et adopté les deux Protocoles additionnels aux 
Conventions de Genève. Ces textes constituent des développements de première importance du droit international humanitaire, notamment en ce qui concerne les règles fixant des limites humanitaires à la conduite des hostilités et celles applicables lors des conflits internes.

Or, ce résultat remarquable paraissait inespéré à l'époque où il a été obtenu, tandis que se déroulaient encore des guerres de libération coloniale et que l'on était en pleine guerre froide. Si cela a néanmoins été possible, c'est bien grâce à l'esprit constructif, voire amical, qui s'était instauré entre les experts et les diplomates qui suivaient ces travaux. En effet, au travers des longues sessions annuelles s'est créée une communauté politique et académique qui s'est soudée au-delà des idéologies, et dont les membres ont progressivement cherché à mieux comprendre la source des divergences, à rechercher des compromis. Plusieurs de ces diplomates ou experts se sont par ailleurs retrouvés entre les sessions de la Conférence diplomatique à l'Institut international de droit humanitaire de San Remo (Italie). Fraîchement créé, cet organe a pu jouer alors un rôle de premier ordre en offrant un cadre sympathique et informel à tous ceux qui donnaient beaucoup d'eux-mêmes pour faire aboutir un projet auquel ils s'attachaient chaque jour davantage.

Konstantin Obradovic était l'une des personnalités les plus marquantes de cette communauté et, jusqu'à sa mort, il avait gardé avec beaucoup des membres de celle-ci des liens d'amitié. Comme plusieurs autres experts, il avait continué de longues années à défendre les textes adoptés et leur cause, en travaillant à leur ratification, en contribuant à leur diffusion, ou en collaborant à leur insertion dans les législations nationales.

On ne saurait par ailleurs rappeler ces liens d'amitié sans mentionner la relation affectueuse que Konstantin Obradovic avait nouée depuis la Conférence diplomatique avec un grand nombre de collaborateurs du CICR. Ce fut par exemple le cas lors de séminaires où il se retrouvait presque "en famille» ou, ces dernières années, avec les délégués du CICR en poste à Belgrade. Il a également contribué à la Revne, qui publie d'ailleurs son dernier article dans le présent numéro.

Dans le cœur de ses innombrables amis, Kosta avait une place particulière. Kosta, dis-je, car le professeur Konstantin Obradovic devenait très rapidement Kosta pour ceux qui l'approchaient, cet ami 
ouvert, direct, "râleur", Kosta au grand cœur qui défendait ses idées avec force et conviction. Que d'heures n'a-t-on pas passées à discuter, à chercher des solutions aux problèmes écrasants qui se sont abattus sur son pays, ou même à refaire le monde.

Très actif dans son pays, Konstantin Obradovic avait contribué avec ardeur à la promotion du droit humanitaire et à la formation dans ce domaine. Il a participé à d'innombrables séminaires et publié de nombreux livres et articles, avec le souci constant de faire comprendre au plus grand nombre comme aux spécialistes les enjeux des droits de l'homme et du droit international humanitaire.

Le démantèlement désordonné de la Yougoslavie, puis l'engrenage de la méfiance, de la haine et de la violence qui s'est installé dans les Balkans, le mépris affiché par beaucoup à l'égard de valeurs pour lesquelles il s'était tant battu, tout cela avait profondément affecté Konstantin Obradovic. L'amertume le gagnait souvent, mais son esprit combatif le poussait à continuer de s'engager pour sortir de ce cercle vicieux, pour défendre les principes essentiels des droits de l'homme et du droit international humanitaire. À cet égard, il avait, pour une courte période, été ministre fédéral adjoint pour les droits de l'homme et pour les minorités, ce qui représentait pour lui un gage d'espoir.

En Yougoslavie, mais aussi à Genève, à San Remo - il était membre de l'Institut de droit humanitaire et un fidèle participant à ses tables rondes - et dans bien d'autres endroits, la voie rocailleuse de Kosta va cruellement manquer, comme dans le cœur de toute la "famille des humanitaires».

Que sa femme et sa famille veuillent bien accepter notre profonde sympathie et nos plus sincères condoléances.

Salut, Kosta !

Yves SANdoz

CICR 


\section{Le Comité international de la Croix-Rouge et la protection des victimes de la guerre}

par François Bugnion, Comité international de la CroixRouge, Genève, 1994/2000, 1444 pages, CHF 59,-, 2 édition inchangée, avec une préface de Cornelio Sommaruga, président du CICR de 1987 à 1999

Fondateur du Mouvement international de la Croix-Rouge et du Croissant-Rouge et promoteur des Conventions de Genève de 1949 , le CICR est présent depuis plus d'un siècle sur la plupart des champs de bataille.

Comment le CICR est-il constitué ?

Quelles sont ses tâches?

Quels sont les principes qui guident son action?

François Bugnion tente d'y répondre en combinant une approche historique et juridique.

Cet ouvrage s'adresse à tous les praticiens de l'action humanitaire et à tous ceux qui se sentent concernés par la protection de la personne humaine au milieu de la guerre.

Veuillez adresser vos commandes au

Comité international de la Croix-Rouge

Centre d'information publique

19, avenue de la Paix

CH-1202 Genève

e-mail:dc_com_cip.gva@icrc.org

fax : ++41227332057 


\section{Information aux auteurs et lignes directrices pour la présentation des manuscrits}

La rédaction de la Revue internationale de la Croix-Rouge invite les lecteurs à lui soumettre des textes sur des sujets en rapport avec l'action, la politique ou le droit humanitaires, ainsi que sur toute question intéressant le Mouvement international de la Croix-Rouge et du Croissant-Rouge (voir la mission de la Revue en page 2 de couverture).

La publication ou non d'un manuscrit et la date de sa parution sont décidées par la rédaction de la Revue. II est notamment tenu compte de l'originalité du travail et de la qualité scientifique de la démarche. Le cas échéant, le texte est soumis à un expert pour avis.

Langue: le manuscrit peut être rédigé en langue française ou anglaise. La Revue publie les textes dans leur langue originale, avec un résumé dans l'autre langue.

Longueur: l'article ne doit pas dépasser 20 pages imprimées (environ 8000 mots). Le compte rendu d'un livre comptera au maximum trois pages imprimées (environ 1 ooo mots). - L'auteur d'un article est invité à en fournir un résumé de 100 à 200 mots, selon la longueur de l'original.

Notes de bas de page: la rédaction invite les auteurs à maintenir le nombre et la longueur des notes de bas de page au minimum nécessaire à la compréhension du texte, à l'identification des sources utilisées et à l'intérêt bibliographique. Les notes sont numérotées de manière consécutive, du début à la fin du texte.

\section{Références bibliographiques :}

a) ouvrage : nom de l'auteur, titre de l'ouvrage, édition (p. ex., $2^{\mathrm{e}}$ édition), éditeur, lieu et année de publication, page(s)

b) article d'un périodique ou d'un ouvrage collectif: nom de l'auteur, titre de l'article et

- périodique: nom du périodique, volume, année, page(s)

- ouvrage collectif: nom des rédacteurs responsables (éds.), titre de la publication, éditeur, lieu et année de publication, page(s)

La rédaction souhaite recevoir les manuscrits sous forme de textes papier accompagnés d'une disquette, ou par e-mail, avec copie papier envoyée par poste ou par fax. L'auteur est invité à envoyer son texte dans un des formats suivants:

\section{WordPro Millennium ou 97}

Word for Windows 6.0, 95 ou 97

Notice biographique: une information biographique de deux ou trois lignes doit accompagner l'article proposé.

La Revue se réserve le droit de réviser la forme des textes. Les manuscrits, publiês ou non, ne sont pas rendus. L'auteur d'un article publié reçoit des tirés-à-part de sa contribution. 


\section{Information for authors and guidelines for the submission of manuscripts}

The International Review of the Red Cross invites readers to submit articles on subjects relating to humanitarian action, law or policy or any issue of interest to the International Red Cross and Red Crescent Movement (see Mission of the Review on the inside front cover).

The decision whether or not to publish a text in the Review is taken by the editor, who also determines the date of publication. The main criteria applied are originality and academic standard. If necessary, an expert opinion will be sought.

Language: Manuscripts should be submitted in either French or English. Texts are published in the original version together with a summary in the other language.

Length: Articles should be no longer than 20 printed pages (around 8,000 words). Book reviews should not exceed three printed pages (around 1,00o words). Authors of articles are kindly asked to provide a summary, comprising 100 to 200 words, depending on the length of the original.

Footnotes: Authors are requested to keep the number and length of footnotes to the minimum necessary for ensuring comprehension of the text, and for indicating the sources used and relevant literature. Footnotes should be numbered consecutively from the beginning of the manuscript.

\section{Bibliographical references :}

(a) Books: author's name, title of book, edition (for example, and ed.), publisher, place and year of publication, and page(s) referred to;

(b) Articles in periodicals or collective publications: author's name, title of the article and, in the case of:

- periodicals: name of periodical, volume, year, and page(s) referred to;

- collective publications: names of editors (eds), title of publication, publisher, place and year of publication, and page(s) referred to.

Manuscripts should be sent to the Review on paper together with a copy on diskette, or by e-mail, in which case a paper copy should also be sent by post or fax. Authors are invited to submit texts in one of the following word-processing programmes:

WordPro Millennium or 97 Word for Windows 6.0, 95 or 97

Biographical notes: Articles submitted for publication should be accompanied by two to three lines of biographical information on the author.

The Review reserves the right to edit articles. Manuscripts, whether or not accepted for publication, will not be returned. Authors of published articles will receive off-prints of their contributions. 